\title{
Designing Cylinders with Constant Negative Curvature
}

\author{
Ulrich Pinkall
}

\begin{abstract}
We describe algorithms that can be used to interactively construct ("design") surfaces with constant negative curvature, in particularly those that touch a plane along a closed curve and those exhibiting a cone point. Both smooth and discrete versions of the algorithms are given.
\end{abstract}

Keywords. Constant Gauss curvature, discrete $K$-surfaces.

\section{Smooth $K$-surfaces}

Here we give a brief introduction to the differential geometry of surfaces with constant negative Gaussian curvature.

\subsection{Overview}

Surfaces with constant negative Gaussian curvature, known as $K$-surfaces, are a classical topic in differential geometry. One reason is that their intrinsic geometry provides a model for the hyperbolic plane. The oldest example known is the so-called pseudosphere, a certain surface of revolution with Gaussian curvature -1 :

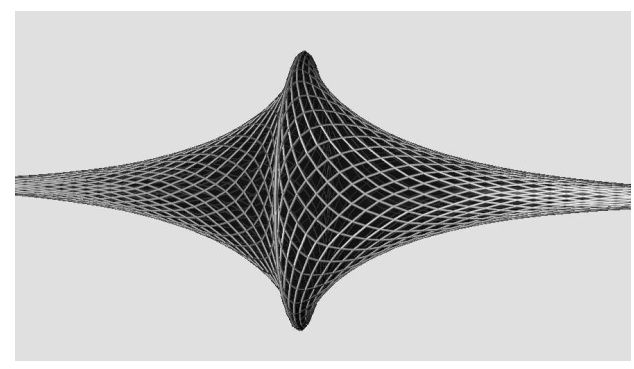

FIGURE 1. The pseudosphere 
Other surfaces of revolution with $K=-1$ come in two types as shown in the pictures below. The first type looks like a series of barrels joined along cuspidal edges:

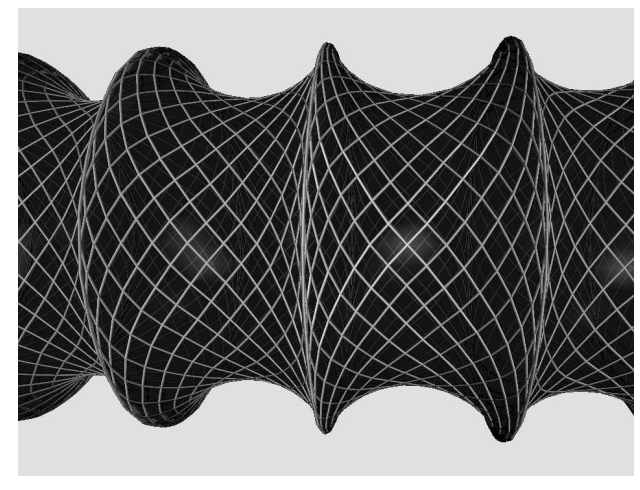

FIGURE 2. $K$-surface of revolution

The other type in addition exhibits singular points where the surface behaves like a cone:

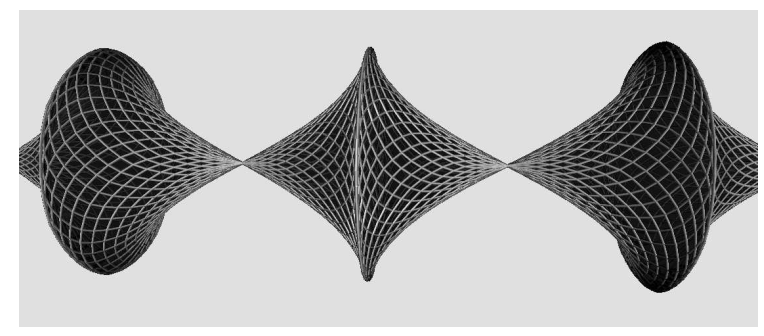

FIGURE 3. $K$-surface of revolution with cone points

By a theorem of Hilbert there is no complete immersed surface with constant negative Gaussian curvature in 3-space. Nevertheless, all the above surfaces can be parametrized by perfectly smooth maps. This is visible in the pictures from the fact that all parameter curves (in fact they are asymptotic lines) extend as smooth curves through the apparent singularities.

Later we will give a precise definition of the regularity conditions we impose on a $K$-surface.

By Hilbert's theorem, globally, singularities have to occur on a $K$-surface. Generically, they come in two types:

- cuspidal edges as in the pictures above,

- swallowtail points, where the cuspidal edges themselves exhibit cusps, as in the picture below.

Cone points do not occur generically. 


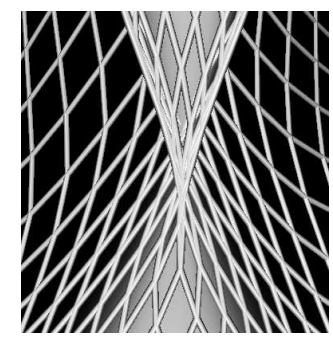

FIGURE 4. Swallowtail singularity

\subsection{The Gauss map of a $K$-surface}

The Gauss map of a $K$-surface has the characterizing property that the images of the asymptotic lines form a Chebyshev net on the 2-sphere. This means that in suitable asymptotic coordinates the Gauss images of the parameter lines are parametrized with constant speed. Visually this implies that the parameter lines form "infinitesimally small parallelograms" on the sphere.

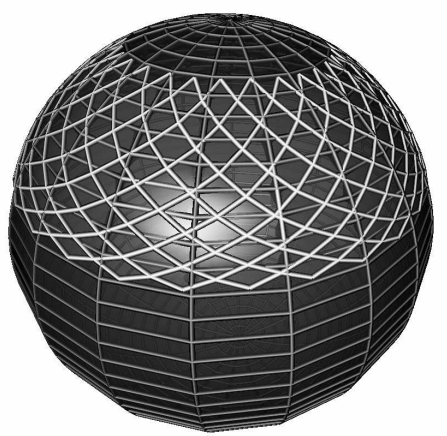

Figure 5. Chebyshev net on $S^{2}$

One way to state this property is that the Gauss map of a $K$-surface is a harmonic map from the plane $\mathbb{R}^{2}$ (endowed with a Lorentz metric where the coordinate lines are lightlike) to the 2-sphere $S^{2}$. Using arbitrary asypmptotic coordinates $u, v$ this is equivalent to the following partial differential equation (subscripts indicate partial derivatives):

$$
N \times N_{u v}=0 .
$$

Switching the coordinates $u, v$ to the coordinates

$$
\begin{aligned}
& x=u+v, \\
& t=u-v,
\end{aligned}
$$


one obtains another physical interpretation of the Gauss map of a $K$-surface. The above PDE for $N$ becomes

$$
N \times\left(N_{x x}-N_{t t}\right)=0
$$

and the images of the lines $t=$ const therefore model the evolution of an elastic string on the sphere $S^{2}$. Think of the continuum limit of a sequence of massive balls coupled by elastic rubber bands.

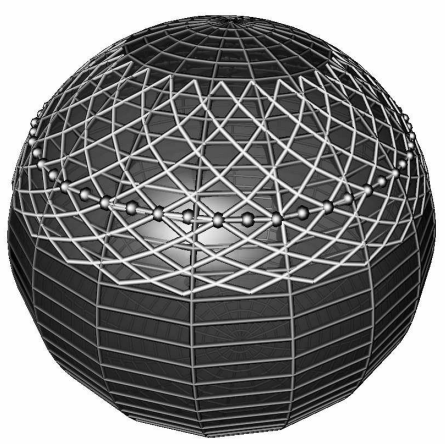

FIGURE 6. Elastic string on $S^{2}$

In the physics literature such a string evolution on $S^{2}$ is referred to as a solution of the "non-linear $\sigma$-model".

\subsection{Reconstruction of a $K$-surface from its Gauss map}

Using asymptotic coordinates $u, v$ one can reconstruct a $K$-surface $f$ from its Gauss map $N$ by solving the following ordinary differential equations:

$$
\begin{aligned}
& f_{u}=N \times N_{u}, \\
& f_{v}=-N \times N_{v} .
\end{aligned}
$$

Using the $x, t$ coordinates introduced above these equations become:

$$
\begin{aligned}
f_{x} & =N \times N_{t}, \\
f_{t} & =N \times N_{x} .
\end{aligned}
$$

\subsection{Precise definition of a $K$-surface}

The most convenient way to give a precise definition of a (not nececessarily immersed) $K$-surface is by using its Gauss map: A map $f: \mathbb{R}^{2} \mapsto \mathbb{R}^{3}$ is called a $K$-surface if there is a smooth map $N: \mathbb{R}^{2} \mapsto S^{2}$ such that the formulas of the previous section apply and

- the partial derivatives $N_{u}$ and $N_{v}$ are nowhere vanishing.

- $N \times N_{u v}=0$. 
Since the second condition implies $\left\langle N_{u}, N_{u}\right\rangle_{v}=0$ and $\left\langle N_{v}, N_{v}\right\rangle_{u}=0$, it is enough to ensure the first condition on some line $u-v=$ const.

It is easy to see that the second condition is equivalent to the exitence of a map $f: \mathbb{R}^{2} \mapsto \mathbb{R}^{3}$ satifying (3) and (4), and the first condition is then equivalent to the map $(f, N): \mathbb{R}^{2} \mapsto \mathbb{R}^{3} \times S^{2}$ being an immersion.

By the formulas of the last section, all parameter lines of $f$ in the $u, v$-coordinates will be curves with non-vanishing derivative (wherever $f$ is an immersion, these curves will be asymptotic lines of $f$ ).

\section{Discrete $K$-surfaces}

Here we explain the discrete differential geometry used to implement a polyhedral version of $K$-surfaces [1, 2]. This should not just be considered as a numerical approximation to the smooth constructions, but as geometrically interesting in its own right.

\subsection{Definition}

A map $f: \mathbb{Z}^{2} \mapsto \mathbb{R}^{3}$ from the integer lattice into 3-space is called a discrete $K$-surface if

- The length $\left|f_{n+1, m}-f_{n, m}\right|$ of a horizontal edge is independent of $m$.

- The length $\left|f_{n, m+1}-f_{n, m}\right|$ of a vertical edge is independent of $n$.

- Each vertex $f_{n, m}$ together with its four neighbors $f_{n-1, m}, f_{n+1, m}, f_{n, m-1}, f_{n, m+1}$ lies in some plane $E_{n, m}$.

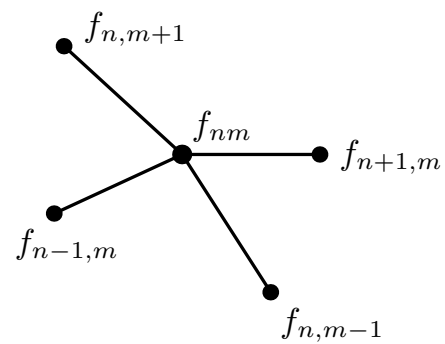

FIgURE 7 . To the definition of a discrete $K$-surface.

The first two properties mean that a discrete $K$-surface is composed out of "skew parallelograms", i.e., quadrilaterals in space where opposite sides have the same length (without being planar). The third property ensures that we have a well defined normal vector $N_{n, m}$ at each vertex $f_{n, m}$, given by the normal vector to the plane $E_{n, m}$. See the pictures in the section on $K$-surfaces. The surfaces depicted there were, in reality, discrete $K$-surfaces. 


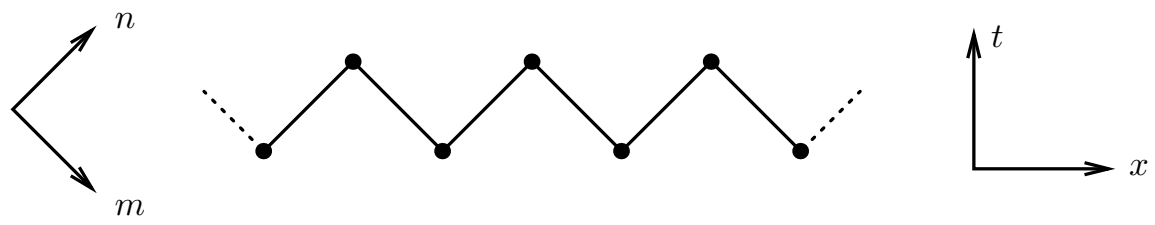

FIGURE 8. To the initial zig-zag.

\subsection{Properties of discrete $K$-surfaces}

The Gauss map of a discrete $K$-surface is always a discrete Chebyshev net in the sense that all quadrilaterals $N_{n, m}, N_{n+1, m}, N_{n+1, m+1}, N_{n, m+1}$ are spherical parallelograms (they admit a $180^{\circ}$-rotation that interchanges opposite points).

A discrete Chebyshev net is completely determined by giving initial data $N_{n, n}$ (time 0 ) and $N_{n+1, n}$ (time 1 ) for all integers $n$. We will refer to such initial data as an "initial zigzag". By successively adding the missing fourth point of a spherical parallelogram (three points of which already have been determined) one can reconstruct a discrete Chebyshev net from an initial zig-zag uniquely.

\subsection{Reconstruction of a discrete $K$-surface from its Gauss map}

One can reconstruct a discrete $K$-surface $f$ from its Gauss map $N$ by solving the following difference equation:

$$
\begin{aligned}
& f_{n+1, m}-f_{n, m}=N_{n, m} \times N_{n+1, m}, \\
& f_{n, m+1}-f_{n, m}=N_{n, m+1} \times N_{n, m} .
\end{aligned}
$$

Here $v \times w$ denotes the cross-product of the vectors $v$ and $w$.

\section{3. $K$-surfaces with a cone point}

\subsection{Smooth case}

A smooth $K$-surface $f$ is said to have a cone point if a whole regular curve $\gamma$ in the parameter domain is mapped to a fixed point. Since the asymptotic lines are always regular by definition, in asymptotic coordinates $u, v$ such a curve can never be tangent to a coordinate line $u=$ const or $v=$ const. Hence, by passing to different asymptotic coordinates we locally may assume that $\gamma$ is equal to the $x$-axis in the coordinates

$$
\begin{aligned}
x & =u+v, \\
t & =u-v .
\end{aligned}
$$

From the formulas describing the reconstruction of a $K$-surface from its Gauss map we see that $f_{x}=0$ implies $N_{t}=0$. This means that at time 0 the moving string on the 2sphere is at rest, i.e., all points have velocity zero. This is then the algorithm to construct cylinders with constant negative Gaussian curvature that exhibit a cone point: 
- Place a closed elastic string $x \mapsto N(x, 0)$ on the 2-sphere and use it as initial data with zero velocity for a string evolution $N: \mathbb{R}^{2} \mapsto S^{2}$.

- Reconstruct the surface $f$ from $N$ as described in the section on the reconstruction of a $K$-surface from its Gauss map.

\subsection{Discrete case}

It is now clear how to construct discrete $K$-surfaces with a cone point: According to the section on Gauss maps of discrete $K$-surfaces one has to start with an initial zig-zag that is stationary, i.e., a fixed point of its discrete evolution. This will occur if and only if every point of the zig-zag at time 1 is situated exactly at the center of the spherical great circle arc connecting the neighboring points at time 0 .

In this case, the points at time -1 obtained by completing the parallelograms will coincide with the corresponding points at time 1. Furthermore the whole Gauss map for negative values of the time will be an exact copy of the Gauss map for positive times.

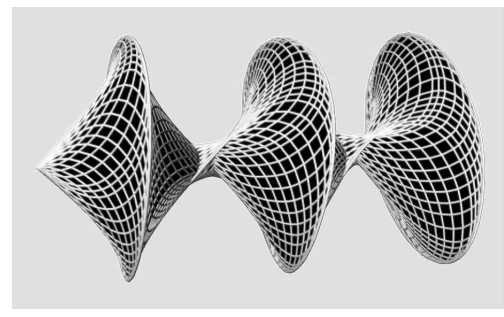

FIGURE 9. A discrete $K$-surface with a cone point.

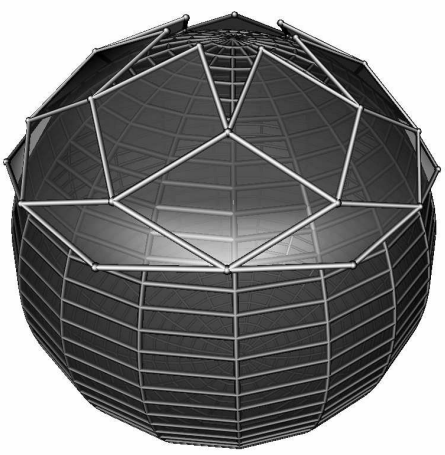

FIGURE 10. Gauss map of a discrete $K$-surface with a cone point. 


\section{4. $K$-surfaces with a planar strip}

\subsection{Smooth case}

A smooth $K$-surface $f$ is said to have a planar strip if a whole regular curve $\gamma$ in the parameter domain is mapped to a fixed point on the 2 -sphere by its Gauss map $N$. Since the asymptotic lines are always regular curves by definition, in asymptotic coordinates $u$, $v$ such a curve $\gamma$ can never be tangent to a coordinate line $u=$ const or $v=$ const. Hence, by passing to different asymptotic coordinates we locally may assume that $\gamma$ is the $x$-axis in the coordinates

$$
\begin{gathered}
x=u+v, \\
t=u-v .
\end{gathered}
$$

From the formulas describing the reconstruction of a $K$-surface from its Gauss map we see that $N(x, 0)$ is a fixed point on $S^{2}$. This is then the algorithm to construct cylinders with constant negative Gaussian curvature with a planar strip:

- Place a closed elastic string in a totally collapsed state at some point on the 2-sphere, provide arbitrary initial velocities $N_{t}(x, 0)$ and use this as initial data for a string evolution $N: \mathbb{R}^{2} \mapsto S^{2}$.

- Reconstruct the surface $f$ from $N$ as described in the section on the reconstruction of a $K$-surface from its Gauss map.

\subsection{Discrete case}

It now clear how to construct discrete $K$-surfaces with a planar strip: One has to start with an initial zig-zag with all $N_{n, n}$ equal to a fixed point $a$ on $S^{2}$.

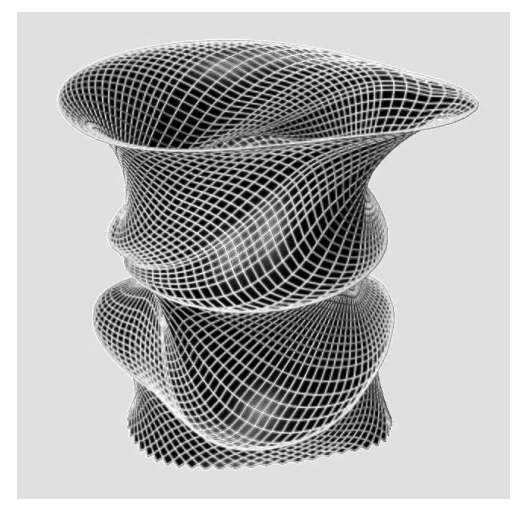

FIGURE 11. A discrete $K$-surface with a planar strip. 


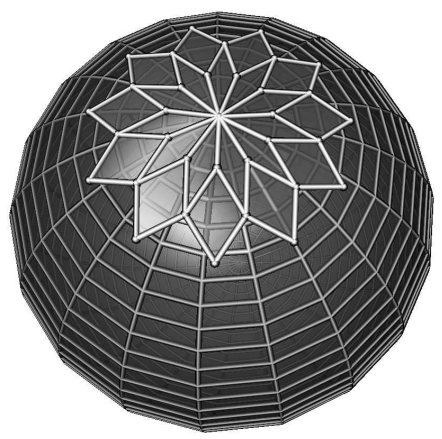

FIGURE 12. Gauss map of a discrete $K$-surface with a planar strip.

According to the section on the reconstruction of a discrete $K$-surface from its Gauss map the corresponding points $f_{n, n}$ will then satisfy

$$
\begin{aligned}
f_{n+1, n}-f_{n, n} & =a \times N_{n+1, n}, \\
f_{n+1, m+1}-f_{n+1, n} & =a \times N_{n+1, n},
\end{aligned}
$$

and therefore

$$
f_{n+1, n+1}-f_{n, n}=2 a \times N_{n+1, n} .
$$

To make a discrete $K$-surface that touches a plane with normal vector $a$ along a prescribed closed polygon $\gamma_{1}, \ldots, \gamma_{m}$ one therefore has to proceed as follows:

- Rotate the polygon by $90^{\circ}$ around $a$ and scale it so that all edge vectors $\gamma_{n+1}-\gamma_{n}$ have length smaller than 2 (this leaves a free parameter for the construction).

- Project the scaled edge vectors $\left(\gamma_{n+1}-\gamma_{n}\right) / 2$ to the unit 2-sphere to obtain the normal vectors $N_{2,1}, \ldots, N_{m+1, m}$.

- Set $N_{n, n}=a$ for all $n$ and use the defined normal vectors as an initial zig-zag for a discrete Chebyshev net.

- Reconstruct the discrete $K$-surface $f$ from its Gauss map $N$.

\section{Software}

Interactive Java webstart applications that implement the algorithms in this paper are available at:

$$
\text { www.math.tu-berlin.de/geometrie/lab }
$$

The material in this paper is also included with these applications, under the help menu.

\section{References}

[1] A.I. Bobenko, U. Pinkall, Discrete surfaces with constant negative Gaussian curvature and the Hirota equation. J. Differential Geom., 43(3):527-611, 1996 
[2] A.I. Bobenko, U. Pinkall, Discretization of Surfaces and Integrable Systems. In: A.I. Bobenko, R. Seiler (eds.) Discrete Integrable Geometry and Physics, Oxford University Press (1999) pp. 3-58, www-sfb288.math.tu-berlin.de/abstractNew/296

Ulrich Pinkall

Institut für Mathematik, MA 3-2

Technische Universität Berlin

Str. des 17. Juni 136

10623 Berlin

Germany

e-mail: pinkall@math.tu-berlin.de 